\title{
THE CAHN-HILLIARD'S EQUATION WITH BOUNDARY NONLINEARITY AND HIGH VISCOSITY *
}

\author{
ROBERT WILLIE ${ }^{\dagger}$
}

\begin{abstract}
The paper studies in less general scales of Banach spaces the dynamics generated by a Cahn-Hilliard type equation in a smooth open bounded domain of any space dimensions. The equation on the boundary satisfy nonlinear conditions. It establishes local well posedness of the problem and a priori uniform on the domain boundedness and existence in the large of the solutions is studied. It also discusses the asymptotic behaviour of the solutions in the form of existence of a global attractor. An adequate notion of upper semicontinuity of the attractor in the limit of high viscosity is considered and the limit attractor is found to correspond to finite dimensional processes. These processes are depicted by limits of the spatial average solutions of the problem.
\end{abstract}

1. Introduction. In this paper, we study the dynamics of solutions in extended scales of Hilbert spaces generated by a fourth order nonlinear parabolic problem

$$
u_{t}+\operatorname{Div}\left(\left(d(x) \nabla^{3}+\nabla\right) u\right)=f(u, \nabla u, \Delta u) \quad \text { in } \Omega, t>0
$$

where $\Omega \subset \mathbb{R}^{N}, N \geq 1$ is an open bounded convex domain with smooth boundary $\partial \Omega=\Gamma$. The equation is supplemented by nonlinear boundary flux and initial conditions of the form

$$
\partial_{\vec{n}_{d}} \Delta u=g(u, \nabla u), \quad \partial_{\vec{n}_{d}} u=0 \quad \text { on } \Gamma \text { and } \quad u(0)=u_{0} \quad \text { in } \Omega,
$$

where $d: \Omega \longmapsto \mathbb{R}^{+} \backslash\{0\}$ is uniformly continuous and denote the viscosity coefficient. The notation

$$
\partial_{\vec{n}_{d}} \cdot=\langle d(x) \nabla \cdot, \vec{n}\rangle \quad \text { with } \vec{n} \text { the exterior unit normal vector at } \Gamma,
$$

denote the boundary derivative operator of the principal spatial differential operator in (1). In the domain the nonlinearity

$$
f(u, \nabla u, \Delta u)=6 u|\nabla u|^{2}+3 u^{2} \Delta u,
$$

complement the physical relevant nonlinearity $\varphi(u)=\Delta\left(u^{3}-u\right)$ in the sense that $f(u, \nabla u, \Delta u)-\Delta u=\varphi(u)$. The boundary nonlinearity is assumed of class $C^{1}$ and satisfy, either no growth assumptions or behavior of the type

$$
\begin{aligned}
& \mid g\left(u_{1}, \nabla u_{1}\right)-g\left(u_{2}, \nabla u_{2}\right) \mid \\
& \leq C\left(1+\sum_{j=1,2}\left|\nabla^{k} u_{j}\right|^{\rho-1}\right)\left|u_{1}-u_{2}\right| \quad \text { for } k=0,1
\end{aligned}
$$

and $\rho \geq 1$ verifying, if $N=4$ then $\rho<\infty$ and if $N \geq 5$ we have

$$
\rho \leq \frac{N+8 \alpha-3}{N-8 \alpha+1} \quad \text { where } 3 / 4>\alpha \geq 1 / 2,
$$

\footnotetext{
*Received August 28, 2003; accepted for publication January 20, 2004.

†Postal Address: P.O. Box 3024, Harare, Zimbabwe (rwillie@maths.uz.ac.zw). Universidad Complutense de Madrid, Facultad de ciencias Matemáticas, Departamento de Matemática Aplicada, 28040 Madrid. España (rwillie@mat.ucm.es). The author during his years as Ph.D student and 5/6 of his D.Sci.Appl.Maths. at La Universidad Complutense de Madrid he studied under financial sponsorship from the institute A.E.C.I. Madrid, Spain.
} 
thither $C \geq 0$ denote any generic constant.

Let us notice that, if in (1)-(2) unit viscosity constant and homogeneous boundary conditions are assumed, the equations are standard of Cahn-Hilliard. Excellent references on the physical background and current state of art of the standard equations (1)-(2) with zero boundary conditions can be found in $[9,13,22,24]$. Although not exhaustive we have provided bibliography where nonlinear parabolic problems with high viscosity (large diffusion) has been studied before. The main objective of this paper is to give a dynamical study of the equations (1)-(2) in less general $[6,12,16,18,19]$ scales of Banach spaces.

Now we can outline the structure of the paper. It is organized as follows. In Section 2, we give the most needed preliminaries and formulate our problem in evolution sense. In Section 3, we prove local well posedness of the problem in extended scales of Hilbert spaces. In Section 4, we study a priori supremum norm boundedness and existence in the large of the solutions of the equations (1)-(2) in $\Omega \subset \mathbb{R}^{N}, N \geq 4$. In Section 5, we discuss the existence of a global attractor of the equation. The affirmative case is found in $N \leq 3$, while the remaining is non conclusive. This is because from the preceeding Section 4, we can not claim $C^{1}(\bar{\Omega})$ regularity and boundedness for all positive time of the solution to the problem, which otherwise would enable us to have suitable reductions on the behaviors of the nonlinearities. The last Section 6 , prove only in the case of the domain of the equation in $N \leq 3$ dimensions, the upper semicontinuity in the limit of high viscosity of the attractor. The limit attractor is of a finite dimensional equation. It attracts limit processes corresponding to the ones defined in the spatial average solutions of the problem.

2. Preliminaries. Throughout the paper, we assume familiarity with the notions of Sobolev spaces $[1,5,14]$ and of general scales of Banach spaces $[2,12,13,19]$ defined by sectorial elliptic differential operators $(A, D(A))$ in $L^{p}(\Omega), 1 \leq p \leq \infty$ such that

$$
X^{\alpha} \cong D\left(A^{\alpha}\right), \quad D(A)=X^{1} \subset W^{m, p}(\Omega), m \geq 1
$$

with continuous inclusions and $0 \leq \alpha \leq 1$. For technical embeddings and interpolations results between these spaces, see $[12,19]$.

2.1. The elliptic operator. Let us now, consider the stationary problem of (1)-(2) and define the bilinear form $a_{d}: H^{2}(\Omega) \times H^{2}(\Omega) \longmapsto \mathbb{R}$ by

$$
a_{d}(u, \varphi)=\int_{\Omega} d(x) \Delta u \Delta \varphi-\int_{\Omega} \nabla u \nabla \varphi .
$$

Then, it is easy to see that (6) is symmetric and continuous. Further, if high viscosity is assumed then we also have coercivity in $H^{2}(\Omega)$. Thus Lax-Milgram's Theorem $[5,14]$ is satisfied. This imply that the bilinear form (6) define an isomorphism $L_{d} u \in H^{-2}(\Omega)$ as follows:

$$
\text { for each } u \in H^{2}(\Omega) \Rightarrow a_{d}(u, \varphi)=\left\langle L_{d} u, \varphi\right\rangle, \quad \forall \varphi \in H^{2}(\Omega) .
$$

Moreover, the restriction $L_{d} u \in L^{2}(\Omega)$ define an elliptic operator $A_{d}: D\left(A_{d}\right) \subset$ $L^{2}(\Omega) \longmapsto L^{2}(\Omega)$ with realization

$$
\begin{gathered}
D\left(A_{d}\right)=\left\{u \in H^{2}(\Omega): A_{d} u=\operatorname{Div}\left(\left(d(x) \nabla^{3}+\nabla\right) u\right) \in L^{2}(\Omega)\right. \\
\text { and } \left.\partial_{\vec{n}_{d}} \Delta u=\partial_{\vec{n}_{d}} u=0 \text { on } \Gamma\right\}
\end{gathered}
$$


and such that $L_{d} u=A_{d} u$ for all $u \in D\left(A_{d}\right)$. It is well known $[12,14,13]$ that $A_{d}$ is a sectorial operator in $L^{2}(\Omega)$ and its resolvent is a compact set as an operator in $L^{2}(\Omega)$. Hence $[12,16,18]$, the extended scales of Hilbert spaces $D\left(A_{d}^{\alpha}\right)=X_{d}^{\alpha}, \alpha \in \mathbb{R}$ are well defined and endowed with the graph norm

$$
\left\|A_{d}^{\alpha} u\right\|_{0}=\|u\|_{\alpha}, \quad \forall u \in X_{d}^{\alpha}, \alpha \in \mathbb{R} .
$$

In particular, we have

$$
X_{d}^{1} \cong D\left(A_{d}\right), \quad X_{d}^{1 / 2} \cong H^{2}(\Omega), \quad X_{d}^{0} \cong L^{2}(\Omega), \quad \text { and } \quad X_{d}^{-1 / 2} \cong H^{-2}(\Omega) .
$$

2.2. The evolution equation. Now we derive a formal evolution equation of the equations (1)-(2) in functional spaces. For this it suffices to observe that in terms of adequate test functions, we can rewrite the Cahn-Hilliard's equation (1)-(2) in the form

$$
\begin{aligned}
u_{t}+L_{d} u & =h(u, \nabla u, \Delta u), \quad t>0 \\
u(0) & =u_{0} \in X_{d}^{\beta},
\end{aligned}
$$

where $-1 / 4 \geq \beta \geq-1 / 2$. The nonlinear form $h(u, \nabla u, \Delta u) \in H^{-2}(\Omega)$ is given and defined by

$$
\langle h(u, \nabla u, \Delta u), \varphi\rangle=\int_{\Omega} f(u, \nabla u, \Delta u) \varphi+\int_{\Gamma} g(u, \nabla u) \varphi,
$$

for any $\varphi \in H^{2}(\Omega)$. In prespective, our study of the dynamics of the equations (1)(2) shall be based on the evolution equation (8). In the following section, we shall investigate its local well posedness.

3. Local well posedness. In this section, we study local existence and uniqueness of solutions of the equations (1)-(2), in the extended scales of Hilbert spaces $X_{d}^{\alpha}, \alpha \in \mathbb{R}$. The main theorem conclude the following.

THEOREM 3.1. Consider the nonlinear evolution problem (8) and assume for domain in space dimensions $N=1,2,3$ that $1 \geq \alpha>3 / 4$ and if $N \geq 4$ that $\alpha \in \mathbb{R}^{+}$ satisfy $1 / 2 \leq \alpha \leq 3 / 4$. Then, the problem (8) is locally well posed and the unique strong solution is given by

$$
u_{d}(t)=e^{-L_{d} t} u_{0}+\int_{0}^{t} e^{-L_{d}(t-s)} h(u(s), \nabla u(s), \Delta u(s)) d s .
$$

In addition satisfy that $u_{d} \in C\left((0, T), X_{d}^{\alpha}\right) \cap C^{1}\left((0, T), X_{d}^{\gamma}\right)$ for any $\gamma<\beta+1$ and the mapping $t \longmapsto u_{d}(t) \in X_{d}^{\alpha}$ is locally Hölder continuous. Moreover, in the sense of distributions and almost everywhere in time, we have the equivalence of the formulations (8) and the equations (1)-(2).

Proof. It suffices to prove that the nonlinear mapping

$$
X_{d}^{\alpha} \ni u \longmapsto h(u, \nabla u, \Delta u) \in X_{d}^{\beta}
$$

for $-1 / 4 \geq \beta \geq-1 / 2$ is locally Lipschitz continuous on bounded subsets. For this we note that from (3) we have

$$
\begin{gathered}
|f(u, \nabla u, \Delta u)-f(w, \nabla w, \Delta w)| \\
\leq 6|u-w||\nabla u|^{2}+\left.6|w||| \nabla u\right|^{2}-|\nabla w|^{2} \mid+ \\
\quad+3\left|u^{2}-w^{2}\right||\Delta u|+3|w|^{2}|\Delta(u-w)| \\
\leq 6|\nabla u|^{2}|u-w|+6|w||\nabla(u-w)|(|\nabla u|+|\nabla w|) \quad+ \\
\quad+3|\Delta u||u+w||u-w|+3|w|^{2}|\Delta(u-w)| .
\end{gathered}
$$


Next we observe that, by virtue of embeddings of the general scales of Banach spaces generated by sectorial elliptic operators in $L^{p}$ spaces into Sobolev's spaces $[12,19]$ we deduce

$$
X_{d}^{\alpha} \subset C^{k}(\bar{\Omega}) \quad \text { for } k=1 \text { if } N=1,2,3 .
$$

Consequently, there exists $L_{f}=L\left(\|\varphi\|_{\infty},\|\nabla \varphi\|_{\infty},\|\Delta \varphi\|_{\infty}\right) \geq 0$ where $\varphi \in X_{d}^{\alpha}$ is either $u$ or $w$ excluding the single validity of the variable, such that the mapping $\varphi \longmapsto f(\varphi, \nabla \varphi, \Delta \varphi) \in L^{2}(\Omega)$ is Lipschitz continuous.

Now in the case $N \geq 4$, we note that the general scales of Banach spaces-Sobolev's type inclusions $[12,19]$ imply that

$$
X_{d}^{\alpha} \subset L^{q}(\Omega) \quad \text { if } q \leq \frac{2 N}{N-8 \alpha} \quad \text { and } \quad X_{d}^{\alpha-1 / 2} \subset L^{r}(\Omega) \quad \text { if } r \leq \frac{2 N}{N+4-8 \alpha}
$$

where $q, r \geq 2$. Moreover $\frac{2}{q}+\frac{2}{r} \leq 1$ thus Hölder's inequality yields that

$$
\begin{gathered}
\int_{\Omega}|f(u, \nabla u, \Delta u)-f(w, \nabla w, \Delta w)|^{2} \\
\leq 2^{4} \times 36\left(\|\nabla u\|_{r}^{4}\|u-w\|_{q}^{2}+\|w\|_{q}^{2}\|\nabla(u-w)\|_{r}^{2} \times\right. \\
\left.\times\left(\|\nabla u\|_{r}^{2}+\|\nabla w\|_{r}^{2}\right)\right)+ \\
+\quad 2^{4} \times 9\left(\|\Delta u\|_{r}^{2}\left(\|u\|_{q}^{2}+\|w\|_{q}^{2}\right)\|u-w\|_{q}^{2}+\right. \\
+\|w\|_{q}^{4}\|\Delta(u-w)\|_{r}^{2} \\
\leq 2^{4} \times 36 C\left(\|u\|_{\alpha}^{2}+\|w\|_{\alpha}^{2}\right)^{2}\|u-w\|_{\alpha}^{2},
\end{gathered}
$$

and hence on bounded subsets of $X_{d}^{\alpha}, 1 / 2 \leq \alpha \leq 3 / 4$ we have the nonlinear mapping (3) is again Lispchitz continuous.

Let us now consider the boundary nonlinearity. We remark that in the case $N=1,2,3$ the Lipschitz continuity is trivial, since $g \in C^{1}$ and from the general scales of Banach spaces-Sobolev type embeddings we have the inclusions (12) are satisfied. It therefore only remains to prove in the case $N \geq 4$. To this end, let $\rho>0$ be as in (5) and set $\frac{1}{\sigma}+\frac{1}{\rho}=1$. Then, using Hölder's inequality and the following

$$
(a+b)^{p} \leq 2^{p}\left(a^{p}+b^{p}\right), \quad p>0, a, b \geq 0
$$

it follows for any $u_{1}, u_{2} \in X_{d}^{\alpha}$, with $1 / 2 \leq \alpha \leq 3 / 4$ satisfying $\left\|u_{j}\right\|_{\alpha} \leq r, j=1,2$ from (4) that

$$
\begin{aligned}
\int_{\Gamma} \mid g\left(u_{1}, \nabla u_{1}\right)-g & \left.\left(u_{2}, \nabla u_{2}\right)\right|^{\sigma} \\
& \leq 2^{3 \sigma} C^{\sigma}\left(\int_{\Gamma}\left|u_{1}-u_{2}\right|^{\sigma}+\int_{\Gamma} \sum_{j=1,2}\left|\nabla^{k} u_{j}\right|^{(\rho-1) \sigma}\left|u_{1}-u_{2}\right|^{\sigma}\right) \\
& \leq 2^{3 \sigma} C^{\sigma}\left(|\Gamma|^{\frac{1}{\sigma}}\right)^{\sigma}\left[1+\sum_{j=1,2}\left(\int_{\Gamma}\left|\nabla^{k} u_{j}\right|^{\rho+1}\right)^{\frac{\rho-1}{\rho+1} \sigma}\right] \times \\
& \times\left(\int_{\Gamma}\left|u_{1}-u_{2}\right|^{\rho+1}\right)^{\frac{\sigma}{\rho+1}}, \quad k=0,1 .
\end{aligned}
$$


In subsequence to the above, we use the inclusions $X_{d}^{\alpha} \subset L^{\rho+1}(\Gamma)$ and since $L^{\sigma}(\Gamma) \subset$ $X_{d}^{\beta},-1 / 4 \geq \beta \geq-1 / 2$, there exists $\mu: \mathbb{R}^{2} \longmapsto \mathbb{R}^{+}$continuous and increasing such that

$$
L_{g}=2^{3} C|\Gamma|^{\frac{1}{\sigma}} \mu\left(\left\|u_{1}\right\|_{\alpha}^{\rho-1},\left\|u_{2}\right\|_{\alpha}^{\rho-1}\right) \geq 0
$$

is the local Lipschitz continuity constant of the nonlinear mapping $X_{d}^{\alpha} \ni u \longmapsto$ $g(u, \nabla u) \in X_{d}^{\beta}$ and the desired result is obtained. Now we can apply abstract semigroup theory results $[12,18,19,20]$ to obtain the conclusions of the theorem including the regularity stated. The rest is evident and our proof is complete.

4. Supremum norm on $\Omega$ smoothness. In this section, we study the existence of a priori uniform in the domain bounds for all positive time of the solution to the problem (8), in the case $N \geq 4$. It is well known [13], that these estimates are available in the case $N \leq 3$ via Sobolev embeddings and $W^{k, p}$ estimates, when in general the nonlinear term in the domain, comes from a polynomial of odd degree

$$
\varphi(\xi)=\sum_{k=0}^{2 q-1} a_{k} \xi^{k}, \quad a_{2 q-1}>0
$$

or when $\varphi$ has a nonnegative primitive function and the representation $f(\xi, \nabla \xi, \Delta \xi)$ $=\Delta \varphi(\xi)$. This is rather simple for $N=1,2$. If $N=3$, a priori estimates guaranteeing existence in the large follow from nontrivial interpolation estimates [24] p.154-158. The method we employ in the proof of the following theorem, is the Moser iteration technique $[3,15,26]$.

THEOREM 4.1. Consider the nonlinear evolution problem (8) and assume in addition that the initial data is uniformly bounded on $\bar{\Omega}$. Assume that

$$
u_{d}\left(t, u_{d}^{0}\right) \in L^{r}(\Gamma) \quad \text { where } r>(N-1)(\rho-1),
$$

is bounded uniformly for $t \in(0, T)$. Then $u_{d}\left(t, u_{d}^{0}\right) \in X_{d}^{\alpha}$ with $\alpha \in \mathbb{R}^{+}$such that $1 / 2 \leq \alpha \leq 3 / 4$, satisfy

$$
\sup _{\Omega}\left|u_{d}\left(t, u_{d}^{0}\right)\right| \leq C, \quad \forall t \geq 0
$$

Proof. We carry out the demostration of Theorem 4.1 in phases. Our first lemma assert the following.

LEMMA 4.2. The solution of the problem (8) satisfy in an almost everywhere sense in time, the differential inequality

$$
\frac{d}{d t} \int_{\Omega}|u|^{r+1}+\left.\left.\beta \int_{\Omega}|\Delta| u\right|^{\frac{r+1}{2}}\right|^{2} \leq c t e \int_{\Omega}|u|^{r+1}+c t e
$$

where cte $\geq 0$ denote some postive constant, $\beta>0$, and $r \geq 1$.

Proof. Consider the problem (8) and in the inner product of $L^{2}(\Omega)$ with $|u|^{r-1} u \in$ $H^{2}(\Omega)$ for $r \geq 1$. Then, integrating by parts we obtain that

$$
\begin{aligned}
\left.\left.\frac{d}{2 d t} \int_{\Omega}|| u\right|^{\frac{r+1}{2}}\right|^{2}+\frac{2 r}{(r+1)^{2}} & \left(\left.\left.\int_{\Omega} d(x)|\Delta| u\right|^{\frac{r+1}{2}}\right|^{2}-\left.\left.2 \int_{\Omega}|\nabla| u\right|^{\frac{r+1}{2}}\right|^{2}\right) \\
\leq & 6 r \int_{\Omega}|\nabla u|^{2}|u|^{r+1}+C \int_{\Gamma}|\nabla u|^{\rho-1}|u|^{r+1}+ \\
+ & C \int_{\Gamma}|u|^{\rho+r}+C \int_{\Gamma}|u|^{r+1} .
\end{aligned}
$$


Now we estimate above (17). Since $r \geq 1$ imply $2 r \geq 2$ and $r+1 \geq 2$, then Hölder and Young's inequalities imply

$$
\begin{aligned}
6 r \int_{\Omega}|\nabla u|^{2}|u|^{r+1} & \leq 6 r^{3}\left(\left.\left.\int_{\Omega}|\nabla| u\right|^{\frac{r+1}{2}}\right|^{\frac{2(r+1)}{r-1}}\right)^{\frac{r-1}{r+1}}\left(\int_{\Omega}|u|^{r+1}\right)^{\frac{2}{r+1}} \\
& \leq\left.\left. 3 r^{4} \int_{\Omega}|\nabla| u\right|^{\frac{r+1}{2}}\right|^{\frac{2(r+1)}{r-1}}+6 r^{3} \int_{\Omega}|u|^{r+1} \\
& \leq 3 r^{4} C\left(\left.\left.\int_{\Omega}|\Delta| u\right|^{\frac{r+1}{2}}\right|^{2}+\left.\left.|\nabla| u\right|^{\frac{r+1}{2}}\right|^{2}\right)^{\frac{\theta(r+1)}{(r-1)}} \times \\
& \times\left(\left.\left.\int_{\Omega}|\nabla| u\right|^{\frac{r+1}{2}}\right|^{2}\right)^{\frac{(1-\theta)(r+1)}{r-1}}+6 r^{3} \int_{\Omega}|u|^{r+1},
\end{aligned}
$$

where the third estimate follows an application of Nirenberg-Gagliardo's inequality $[12,19]$ with $\theta=\frac{N}{2}-\frac{N(r-1)}{2(r+1)}$.

Now successively we use Young's inequality in [5] pp.56, let $\frac{1}{s}+\frac{1}{s^{\prime}}=1$ then

$$
a b \leq \varepsilon a^{s}+C_{\varepsilon} b^{s^{\prime}}, \quad \text { with } a, b \geq 0,0<\varepsilon<1, C_{\varepsilon}=\varepsilon^{-\frac{1}{s-1}} .
$$

Let $\varepsilon=\varepsilon(\delta)>0$ be adequately chosen with $\delta>0$ sufficiently small. Then it follows that

$$
\begin{aligned}
6 r \int_{\Omega}|\nabla u|^{2}|u|^{r+1} & \leq 2 \delta \int_{\Omega}\left(\left.\left.|\Delta| u\right|^{\frac{r+1}{2}}\right|^{2}+\left.\left.|\nabla| u\right|^{\frac{r+1}{2}}\right|^{2}\right) \\
& +6 r^{3} \int_{\Omega}|u|^{r+1}+C_{\delta} .
\end{aligned}
$$

Similiarly, if $\sigma=r+1-\frac{(r-1)(\rho-1)}{2}>0$. We have using Hölder and Young's inequalities that

$$
\begin{aligned}
C \int_{\Gamma}|\nabla u|^{\rho-1}|u|^{r+1} & \leq C\left(\left.\left.\int_{\Gamma}|\nabla| u\right|^{\frac{r+1}{2}}\right|^{\frac{2(r+1)}{r-1}}\right)^{\frac{(r-1)(\rho-1)}{2(r+1)}}\left(\int_{\Gamma}|u|^{r+1}\right)^{\frac{\sigma}{r+1}} \\
& \leq\left.\left.\rho r C \int_{\Gamma}|\nabla| u\right|^{\frac{r+1}{2}}\right|^{\frac{2(r+1)}{r-1}}+r C \int_{\Gamma}|u|^{r+1} \\
& \leq \rho r C\left(\left.\left.\int_{\Omega}|\Delta| u\right|^{\frac{r+1}{2}}\right|^{2}+\left.\left.|\nabla| u\right|^{\frac{r+1}{2}}\right|^{2}\right)^{\frac{\theta(r+1)}{(r-1)}} \times \\
& \times\left(\left.\left.\int_{\Omega}|\nabla| u\right|^{\frac{r+1}{2}}\right|^{2}\right)^{\frac{(1-\theta)(r+1)}{r-1}}+r C \int_{\Gamma}|u|^{r+1}
\end{aligned}
$$

where the last estimates follow from Nirenberg-Gagliardo' s inequality $[12,19]$ with $\theta=\frac{N}{2}-\frac{(N-1)(r-1)}{2(r+1)}$. Thus, a repeated application of Young's inequality (18) yield

$$
\begin{aligned}
C \int_{\Gamma}|\nabla u|^{\rho-1}|u|^{r+1} & \leq 2 \delta \int_{\Omega}\left(\left.\left.|\Delta| u\right|^{\frac{r+1}{2}}\right|^{2}+\left.\left.|\nabla| u\right|^{\frac{r+1}{2}}\right|^{2}\right) \\
& +r C \int_{\Gamma}|u|^{r+1}+C_{\delta}
\end{aligned}
$$

But, by virtue of Nirenberg-Gagliardo's inequality in [15] for $L^{p}$ functions on $\Gamma$ and Young's (18) we find if $\varepsilon(\delta)=\frac{\delta}{r C}$ that

$$
r C \int_{\Gamma}|u|^{r+1} \leq\left.\left.\delta \int_{\Omega}|\nabla| u\right|^{\frac{r+1}{2}}\right|^{2}+r C \int_{\Omega}|u|^{r+1} .
$$


Consequently

$$
\begin{aligned}
C \int_{\Gamma}|\nabla u|^{\rho-1}|u|^{r+1} & \leq 3 \delta \int_{\Omega}\left(\left.\left.|\Delta| u\right|^{\frac{r+1}{2}}\right|^{2}+\left.\left.|\nabla| u\right|^{\frac{r+1}{2}}\right|^{2}\right) \\
& +r C \int_{\Omega}|u|^{r+1}+C_{\delta}
\end{aligned}
$$

It only remains the third term in (17). Thanks to references in [26] we have, if $r \geq 1$ is as in (15) then there exists $\theta>0$ such that $r=(N-1)(\rho-1)+\theta$ and Nakao-Hölder's inequality imply

$$
\int_{\Gamma}|u|^{\rho+r} \leq\left(\int_{\Gamma}|u|^{\frac{(r+1)(N-1)}{N-2}}\right)^{\Theta_{1}}\left(\int_{\Gamma}|u|^{r}\right)^{\Theta_{2}}\left(\int_{\Gamma}|u|^{r+1}\right)^{\Theta_{3}}
$$

where

$$
\begin{gathered}
\Theta_{1}=\frac{(N-2)(\rho-1)}{(N-1)(\rho-1)+\theta}, \quad \Theta_{2}=\frac{\rho-1}{(N-1)(\rho-1)+\theta} \\
\text { and } \Theta_{3}=\frac{\theta}{(N-1)(\rho-1)+\theta} .
\end{gathered}
$$

Thus the continuity of the inclusions $H^{1}(\Omega) \subset L^{\Theta}(\Gamma)$ with $\Theta=\frac{2(N-1)}{N-2}$ conclude that

$$
\begin{aligned}
\int_{\Gamma}|u|^{\rho+r} & \leq C\left(\left.\left.\int_{\Omega}|\nabla| u\right|^{\frac{r+1}{2}}\right|^{2}+\int_{\Omega}\left(|u|^{\frac{r+1}{2}}\right)^{2}\right)^{\frac{(N-1) \Theta_{1}}{N-2}}\left(\int_{\Gamma}|u|^{r}\right)^{\Theta_{2}} \times \\
& \times\left(\int_{\Gamma}|u|^{r+1}\right)^{\Theta_{3}} .
\end{aligned}
$$

Since $\frac{(N-1) \Theta_{1}}{N-2}+\Theta_{3}=1$, we can use Young's inequality (18) and the hypothesis (15) imply that

$$
\begin{gathered}
r C \int_{\Gamma}|u|^{\rho+r} \leq \delta\left(\left.\left.\int_{\Omega}|\nabla| u\right|^{\frac{r+1}{2}}\right|^{2}+\int_{\Omega}\left(|u|^{\frac{r+1}{2}}\right)^{2}\right)+ \\
+r^{\frac{1}{\Theta_{3}}} C^{\frac{2(\rho-1)}{\theta}} \int_{\Gamma}|u|^{r+1}
\end{gathered}
$$

Now arguing as after $(20)$ we obtain $C_{\varepsilon(\delta)}=\frac{C}{\varepsilon(\delta)}>0$ for $\varepsilon(\delta)=\left(r^{\frac{1}{\Theta_{3}}} C^{\frac{2(\rho-1)}{\theta}}\right)^{-1} \delta$ such that

$$
\begin{gathered}
r C \int_{\Gamma}|u|^{\rho+r} \leq 2 \delta\left(\left.\left.\int_{\Omega}|\nabla| u\right|^{\frac{r+1}{2}}\right|^{2}+\int_{\Omega}\left(|u|^{\frac{r+1}{2}}\right)^{2}\right)+ \\
+r^{\frac{2}{\Theta_{6}}} C^{\frac{2(\rho-1)}{\theta}} \int_{\Omega}|u|^{r+1}
\end{gathered}
$$

Grouping all the above in (17) and without loss of exactness we find the differential inequality

$$
\frac{d}{d t} \int_{\Omega}|u|^{r+1}+\left.\left.\beta \int_{\Omega}|\Delta| u\right|^{\frac{r+1}{2}}\right|^{2} \leq(r C)^{\sigma_{1}} \int_{\Omega}|u|^{r+1}+r C
$$


where $\beta=\inf _{\Omega} d(x)-c(\delta)>0, \sigma_{1}=\sigma\left(N, \rho, \Theta_{j}, \theta\right)>0, j=1,2,3$ and the desired result is proved.

Lemma 4.3. Let $r_{i}=2^{i}, i \geq 1$ and define $y_{i}(t)=\int_{\Omega}|u|^{r_{i}+1}$ then

$$
y_{i}(t) \leq C\left(r_{i} C\right)^{\sigma}\left(K^{\left(r_{i-1}+1\right) s_{i}}+\left(\sup _{t \in(0, T)} y_{i-1}(t)\right)^{s_{i}}\right),
$$

for some $\sigma>0$, where $s_{i}=\frac{r_{i}+1}{r_{i-1}+1}$ and $K=K\left(\left\|u_{0}^{\varepsilon}\right\|_{\infty}\right) \geq 0$.

Proof. Consider the differential inequality (26). If $r_{i}=2^{i}, i \geq 1$ define

$$
\Theta_{i}=\frac{2\left(r_{i}+1\right)}{N\left(r_{i}+1\right)-(N-2)\left(r_{i-1}+1\right)} \quad \text { and } \quad \Theta_{i}^{\prime}=1-\Theta_{i} .
$$

Then, Hölder's inequality and Sobolev's embeddings conclude

$$
\begin{aligned}
\int_{\Omega}|u|^{r_{i}+1} & \leq\left(\int_{\Omega}|u|^{\frac{N\left(r_{i}+1\right)}{N-2}}\right)^{\Theta_{i}^{\prime}}\left(\int_{\Omega}|u|^{r_{i}+1}\right)^{\Theta_{i}} \\
& \leq C\left(\int_{\Omega}\left(|u|^{\frac{r_{i}+1}{2}}\right)^{2}+\left.\left.\int_{\Omega}|\nabla| u\right|^{\frac{r_{i}+1}{2}}\right|^{2}\right)^{\frac{N \Theta_{i}^{\prime}}{N-2}}\left(\int_{\Omega}|u|^{r_{i}+1}\right)^{\Theta_{i}} .
\end{aligned}
$$

Further, $\frac{N \Theta_{i}^{\prime}}{N-2}<1$ using Young's inequality (18) we get

$$
\begin{array}{r}
\left(r_{i} C\right)^{\sigma_{1}} \int_{\Omega}|u|^{r_{i}+1} \leq \delta\left(\int_{\Omega}\left(|u|^{\frac{r_{i}+1}{2}}\right)^{2}+\left.\left.\int_{\Omega}|\nabla| u\right|^{\frac{r_{i}+1}{2}}\right|^{2}\right)+ \\
+\left(r_{i} C\right)^{\sigma}\left(\int_{\Omega}|u|^{r_{i-1}+1}\right)^{s_{i}}
\end{array}
$$

where $s_{i}=\frac{r_{i}+1}{r_{i-1}+1}$ and $\sigma=\sigma_{1}(N+2) / 2 \geq \sigma_{1}\left(N r_{i-1}+r_{i}+2\right) /\left(r_{i}+2\right)$. Consequently in (26) on setting $y_{i}(t)=\int_{\Omega}|u|^{r_{i}+1}$ we obtain

$$
\frac{d y_{i}}{d t}+\beta y_{i} \leq\left(r_{i} C\right)^{\sigma}\left(y_{i-1}+1\right)^{s_{i}}
$$

for some $\beta=\beta(C, \eta)>0$, without loss of generality $C \geq 1$ and we have used [23] Theorem 11.11. It is easy to see, the inequality solve as

$$
y_{i}(t) \leq C\left(r_{i} C\right)^{\sigma}\left(y_{i}(0)+1+\left(\sup _{t \in(0, T)} y_{i-1}(t)\right)^{s_{i}}\right) .
$$

But, by hypothesis on the initial data there is $K=K\left(\left\|u_{0}^{\varepsilon}\right\|_{\infty}\right)>0$ such that $y_{i}(0)+1 \leq$ $K^{\left(r_{i-1}+1\right) s_{i}}$. Hence we obtain $(27)$ and the proof of the lemma is complete.

LEMMA 4.4. The general iteration scheme of (27) for $i=k \gg 1$ large is bounded above independent of $k \gg 1$ in terms of

$$
\sup _{t \in(0, T)} \int_{\Omega}|u|^{2}
$$

consequently, the solution of the problem (8) satisfy (16). 
Proof. Consider the iterative inequality (27) and observe that if $i=1$, then

$$
y_{1}(t) \leq C(2 C)^{\sigma} K^{2 s_{1}}+C(2 C)^{\sigma}\left(\sup _{t \in(0, T)} \int_{\Omega}|u|^{2}\right)^{s_{1}} .
$$

Inductively the iterative process, for $i=2$ and using the elementary inequality (13) which we rewrite here for convenience

$$
(a+b)^{p} \leq 2^{p}\left(a^{p}+b^{p}\right) \quad \text { for } \quad a, b \geq 0, \quad p>0,
$$

we get that

$$
\begin{aligned}
y_{2}(t) & \leq(2 C)^{1+s_{2}}(2 C)^{2 \sigma+2 \sigma s_{2}} K^{2 s_{1} s_{2}}+ \\
& +(2 C)^{1+s_{2}}(2 C)^{2 \sigma+2 \sigma s_{2}}\left(\sup _{t \in(0, T)} \int_{\Omega}|u|^{2}\right)^{s_{1} s_{2}} .
\end{aligned}
$$

Similarly as for (31) we conclude in the third iteration ,

$$
\begin{aligned}
y_{3}(t) & \leq(2 C)^{1+2 s_{3}+2 s_{2} s_{3}}(2 C)^{3 \sigma+2 \sigma s_{3}+\sigma s_{2} s_{3}} K^{2 s_{1} s_{2} s_{3}}+ \\
& +(2 C)^{1+2 s_{3}+2 s_{2} s_{3}}(2 C)^{3 \sigma+2 \sigma s_{3}+\sigma s_{2} s_{3}}\left(\sup _{t \in(0, T)} \int_{\Omega}|u|^{2}\right)^{s_{1} s_{2} s_{3}} .
\end{aligned}
$$

We now deduce, in general, the inductive process, for arbitrary $i=k \geq 3$ furnishes that

$$
\begin{aligned}
y_{k}(t) & \leq(2 C)^{1+2 s_{k}+2 s_{k-1} s_{k}+\ldots+2 s_{2} s_{3} \ldots s_{k}} \times \\
& \times(2 C)^{k \sigma+(k-1) \sigma s_{k}+\ldots+\sigma s_{2} s_{3} \ldots s_{k}} K^{2 s_{1} s_{2} \ldots s_{k}}+ \\
& +(2 C)^{1+2 s_{k}} \quad+2 s_{k-1} s_{k}+\ldots+2 s_{2} s_{3} \ldots s_{k} \times \\
& \times(2 C)^{k \sigma+(k-1) \sigma s_{k}+\ldots+\sigma s_{2} s_{3} \ldots s_{k}}\left(\sup _{t \in(0, T)} \int_{\Omega}|u|^{2}\right)^{s_{1} s_{2} s_{3} \ldots s_{k}} \\
& \leq(2 C)^{2 A_{k}}(2 C)^{\sigma B_{k}} K^{2 \chi_{k}}+(2 C)^{2 A_{k}}(2 C)^{\sigma B_{k}}\left(\sup _{t \in(0, T)} \int_{\Omega}|u|^{2}\right)^{\chi_{k}}
\end{aligned}
$$

where $\chi_{k}=s_{k} \ldots s_{1} \leq \frac{r_{k}+1}{2}$ and

$$
\begin{aligned}
& A_{k}=1+s_{k}+s_{k} s_{k-1}+\ldots+s_{k} s_{k-1} \ldots s_{1} \leq\left(r_{k}+1\right) \sum_{i=0}^{\infty} \frac{1}{r_{i}+1}, \\
& B_{k}=k+(k-1) s_{k}+(k-2) s_{k} s_{k-1}+\ldots+s_{k} s_{k-1} \ldots s_{1} \leq\left(r_{k}+1\right) \sum_{i=1}^{\infty} \frac{i}{r_{i}+1} .
\end{aligned}
$$

Since $r_{i}=2^{i}$ we have the above series converge and it follows

$$
\begin{aligned}
y_{k}(t) & \leq\left((2 C)^{2 \omega_{1}}(2 C)^{\sigma \omega_{2}} K+(2 C)^{2 \omega_{1}}(2 C)^{\sigma \omega_{2}} \sup _{t \in(0, T)}\left(\int_{\Omega}|u|^{2}\right)^{1 / 2}\right)^{r_{k}+1} \\
& \leq\left((2 C)^{2 \omega_{1}}(2 C)^{\sigma \omega_{2}} K\left(\sup _{t \in(0, T)}\left(\int_{\Omega}|u|^{2}\right)^{1 / 2}+1\right)\right)^{r_{k}+1}
\end{aligned}
$$


where $\omega_{1}=\sum_{i=1}^{\infty} \frac{1}{r_{i}+1}$ and $\omega_{2}=\sum_{i=1}^{\infty} \frac{i}{r_{i}+1}$.

Passing the power index $r_{k}+1$ to the left and taking up limits as $k \rightarrow \infty$,we obtain

$$
\begin{aligned}
\sup _{\Omega}\left|u\left(t, u_{0}\right)\right| & \leq \lim _{k \rightarrow \infty}\left(\int_{\Omega}|u|^{r_{k}+1}\right)^{\frac{1}{r_{k}+1}} \\
& \leq(2 C)^{2 \omega_{1}}(2 C)^{\sigma \omega_{2}} K\left(\sup _{t \in(0, T)}\left(\int_{\Omega}|u|^{2}\right)^{1 / 2}+1\right)
\end{aligned}
$$

for all $t \geq 0$. This therefore complete the proof of our lemma.

In conclusion, combining all the above lemmas we obtain the proof of our core Theorem 4.1.

Let us remark that, even though the solution to the equation (8) and its time derivative are spatially differentiable almost everywhere in the function spaces, see Theorem 3.1 and we can consider the problem defined by $\varphi=\nabla u$. Thus seems likely that under appropriate boundedness hypotheses of the data and of the solution of the new auxiliary problem, test functions of the form $|\nabla u|^{r-1} \nabla u, r \geq 1$ will yield an integral differential inequality of the type (26) for gradient $\nabla u$ of the solution. However, in this approach there appear an important sign problem in the initial integral differential inequality of the form (17), which make the method of the proof of Theorem 4.1 obsolescent for proving a priori first order continuously differentiable smoothness on $\bar{\Omega}$ and existence in the large of the solutions to the problem (8).

5. The universal attractor. In this section, we discuss the asymptotic behaviour for long time of the solutions to our problem (1)-(2) in the form of existence of a global attractor. Such dynamical structures for equations of pattern formation have been studied before $[7,8,9,17,24]$. Our aim here is to provide an analogueous study but in extended scales of Hilbert spaces. The main theorem of the section assert the following.

THEOREM 5.1. The nonlinear semigroup generated by the evolution equation (8) in the scales for $1 \geq \alpha>3 / 4$ has a global compact attractor $\mathcal{A}_{d} \subset X_{d}^{\alpha}$, and the limit set $\omega\left(u_{0}\right) \subset \mathcal{A}_{d}$.

Proof. It suffices to observe that the local semigroup generated by (8) is bounded dissipative independent of the initial data in bounded subsets $B \subset X_{d}^{\alpha}, 1>\alpha>3 / 4$, uniformly in $t>0$.

Thus using [19] Theorem 7.9, we get the orbit $\left\{u_{d}\left(t, u_{0}\right): t>0\right\}$ is relatively compact in $X_{d}^{\alpha}, 1>\alpha>3 / 4$. Moreover, using [10] or [8] Theorem 1 we conclude there is a subset $\mathcal{A}_{d} \subset X_{d}^{\alpha}, 1>\alpha>3 / 4$, attracting each point in $B$. This is true, since using (10) we have the semigroup is point dissipative. As the $\omega$-limit set $\omega\left(u_{0}\right)=\cap_{t \geq \tau} \overline{\left\{u_{d}\left(t, u_{0}\right)\right\}}$, for any $\tau>0$, is connected, compact, invariant and minimal attractive set, we must have it contained in $\mathcal{A}_{d}$. This completes the proof of the theorem. a Let us comment that in the case of homogeneous boundary conditions to (8), the attractor has a special form $[8,9]$

$$
\mathcal{A}_{d}=\left\{\varphi \in X_{d}^{\alpha}:\left|\varphi_{\Omega}\right| \leq b, b>0\right\}, \quad \text { where } \quad \varphi_{\Omega}=\frac{1}{|\Omega|} \int_{\Omega} \varphi
$$

and is not an attractor in the sense of [10] p. 17 as the stationary problem contains an arbitrary constant function, hence unbounded in $X_{d}^{\alpha}$. 
Technically the existence of an attractor in the case $N \geq 4$ is delicate. This is due to insufficient uniform continuously differentiable smoothness in the closed domain and boundedness up to maximum time of existence of solutions to the evolution equation (8). The need of these is that they render controllability in the sense of estimates on the nonlinearities. For in an alternative approach, we concretely compute the primitive function to the complement of the physical relevant nonlinearity in the domain, and associate to the equation the energy functional

$$
\begin{aligned}
J_{d}(u) & =\frac{1}{2}\left(\int_{\Omega} d(x)|\Delta u|^{2}-\int_{\Omega}|\nabla u|^{2}\right) \\
& -\int_{\Omega}\left(|u \nabla u|^{2} \Delta u+u^{3} \nabla u|\Delta u|^{2}\right)-\int_{\Gamma} G(u, \nabla u),
\end{aligned}
$$

where

$$
G(u, \nabla u)=\int_{0}^{\nabla u} \int_{0}^{u} g(r, s) d r d s .
$$

Then we show (32) is of Liapunov for the evolution process in (8). Thus, we would conclude of immediate bounded dissipativeness of the solution in $X_{d}^{1 / 2}$ and the method in [7] ( also has been used in [28]) is applicable for the existence of an attractor. The approach of [7] in the otherwise fails due to the lack of an adequate notion of dissipative conditions for strongly dependent on arguments vector valued nonlinearities of the type to our problem. It is the point dissipative property of the semigroup generated by the dynamical system of the equation that we cannot assert. Thus, in concrete we do not know whether the nonlinear semigroup generated by solutions to (8) has an attractor when the equations (1)-(2) are defined in a smooth open bounded domain $\Omega \subset \mathbb{R}^{N}$ for $N \geq 4$ space dimensions.

6. Convergence of Attractors. The upper semicontinuity of attractors in the limit case of high viscosity has been a theme of study in the last two and half decades. The main problems so far exhausted have been second order parabolic and hyperbolic equations. Of most recent, situations have included nonlinear boundary conditions, localized high viscosity in the domain and the asymptotic behavior of the inertial manifold, see for examples the references at the end of paper.

We now study the convergence of attractors as $d=\inf _{\Omega}\{d(x)\} \rightarrow \infty$ in the equation (8). This limit phenomenon of high viscosity in the equation has the effect of stablizing all spatial inhomogeneities. In such a way that there is homogenization to a constant function in $\Omega$ of solutions to the problem. This conclusion holds from previously known results in second order nonlinear parabolic problems. It is reconfirmed here in the subsequent lines for a fourth order equation (1)-(2). In particular, if homogeneous boundary conditions in (2) are considered, the limit solution describe limit processes of the mass conservation in time property, i.e

$$
\bar{u}_{d}(t)=\int_{\Omega} u_{d}=\bar{u}_{d}^{0}, \quad t \geq 0 .
$$

Our first theorem of the section state the following.

TheOREM 6.1. Consider the nonlinear evolution problem (8) and let $u_{d}(t) \in X_{d}^{\alpha}$ for any $\alpha<1$ a.e in time, denote its solution. Denote by

$$
u_{\Omega}=|\Omega|^{-1} u \chi_{\Omega} \in L^{1}(\Omega), \quad \text { where } \quad \chi_{X}= \begin{cases}1 & \text { if } x \in X \\ 0 & \text { otherwise }\end{cases}
$$


and assume the differential equation

$$
\dot{u}_{\Omega}=h_{\Omega}(u), \quad u_{\Omega}(0)=u_{\Omega}^{0} \in \mathbb{R}
$$

where $h_{\Omega}(u)=\frac{|\Gamma|}{|\Omega|} g(u, 0) \chi_{\Gamma} \in L^{1}(\Gamma)$ is satisfied. Then

$$
\lim _{d \rightarrow \infty} \sup _{t>0} \int_{\Omega}\left|\nabla u_{d}\right|^{2}=0 \quad \text { and } \quad u_{d}\left(t, u_{d}^{0}\right) \rightarrow u_{\Omega}\left(t, u_{\Omega}^{0}\right)
$$

strongly in $L^{\infty}\left(0, T, X_{d}^{\alpha}\right)$ for any $\alpha<1$ as $d=\inf d(x) \rightarrow \infty$.

Proof. Let us denote by $\lambda_{2}^{N}(d) \in \sigma\left(A_{d}\right)$ the first non zero eigenvalue of the operator (7). Then, using [21] Theorem 3.6 we have that

$$
\lambda_{2}^{N}(d) \geq d \mu_{2}^{N}
$$

where $\mu_{2}^{N}$ is the second eigenvalue of $-\Delta$ subject to Neumann homogeneous boundary conditions.

Now, consider the variation of constants formula (10) and since on bounded subsets of the scales of spaces we have the nonlinear map (11) is local Lipschitz continuous, it follows that

$$
\begin{aligned}
\left\|u_{d}(t)\right\|_{\alpha} & \left.\leq M e^{-d \mu_{2}^{N} t} t^{-\vartheta} \| u_{d}^{0}\right) \|_{\alpha}+ \\
& +M L_{h} \sup _{0 \leq s \leq t}\left\|u_{d}(s)\right\|_{\alpha} \int_{0}^{t} e^{-d \mu_{2}^{N}(t-s)}(t-s)^{-\vartheta} d s \\
& \leq M L_{h} \sup _{0 \leq s \leq t}\left\|u_{d}(s)\right\|_{\alpha} \int_{0}^{\infty} e^{-d \mu_{2}^{N} t} t^{-\vartheta} d t
\end{aligned}
$$

where $\vartheta=\alpha-\beta>0$, and $L_{h}>0$ denote the Lipschitz constant. Thus, we easily see that

$$
\left\|u_{d}(t)\right\|_{\alpha} \rightarrow 0, \quad \text { uniformly on } \mathbb{R}^{+} \text {as } d \rightarrow \infty .
$$

Therefore, using [23] Theorem 11.11 we conclude the first statement and the lower semicontinuity of the $H^{1}(\Omega)$ norm yields $u_{d}(t) \rightarrow u(t) \in \mathbb{R}$ strongly in $X_{d}^{\alpha}$ uniformly on any finite time interval, as $d \rightarrow \infty$. Otherwise, if $T=\infty$ since by uniqueness of the limit $u(t)=u_{\Omega}(t)$ verify the equation $(34)$, then $u_{\Omega}(t)$ is not necessarily bounded.

Next our final theorem state the following.

THEOREM 6.2. Consider the ordinary differential problem (34) and assume the dissipative hypothesis

$$
\limsup _{|u| \rightarrow \infty} \frac{h_{\Omega}(u)}{u}<0, \quad \text { hold. }
$$

Then, the semiflow defined by (34) has a global compact attractor $\mathcal{A} \subset \mathbb{R}$. Moreover, if $N \leq 3$ then it is satisfied that

$$
\lim _{d \rightarrow \infty} \delta\left(\mathcal{A}_{d}, \mathcal{A}\right)=\lim _{d \rightarrow \infty} \sup _{u_{d} \in \mathcal{A}_{d}} \inf _{u_{\Omega} \in \mathcal{A}}\left\|u_{d}\left(t, u_{d}^{0}\right)-u_{\Omega}\left(t, u_{\Omega}^{0}\right)\right\|_{\alpha}=0
$$

where $1 \geq \alpha>3 / 4$. In other words, the attractors of the equations (8) and (34) are upper semicontinuous in the limit of high viscosity. 
Proof. The first part of the theorem is standard. In fact (34) is point dissipative from which the conclusion follows. The second part is obtained using Theorem 6.1 and $[10]$ p. 165, Sect. 4.10.2.

Acknowledgement. The author would like to sincerely thank the referees for stimulating and interesting reports. Their indications, although apparently seemed minor were very important. Consequently, the suggestions yielded numerous improvements in the redaction of this final version of the paper. In particular, for also bringing to his attention the paper by Li Zhong, Journal of Differential Equations, 149 pp.191210, 1998, where the most recent results on the CH-equation were said to have been reported and the new book by G.Sell \& Y.You [22]. Finally, special thanks are due to my family.

\section{REFERENCES}

[1] R. A. Adams, Sobolev spaces, Academic Press, New York, 1985.

[2] H. Amann, Parabolic Evolution Equations and Nonlinear Boundary Conditions, Journal of Differential Equations, 72 (1988), pp. 201-269.

[3] N. D. Alikakos, $L^{p}$ bounds of solutions of Reaction-Diffusion Equations, Communications in Partial Differential Equations, 4:8 (1979), pp. 827-868.

[4] J. Arrieta, A. R. Bernal And A. N. Carvalho, Upper semicontinuity of attractors for parabolic problems with localized large diffusion and nonlinear boundary conditions, Journal of Differential Equations, 168 (2000), pp. 33-59.

[5] H. BRÉzis, Análisis funcional Alianza Universidad Textos, Masson Editeur, de Paris 1983.

[6] A. Carvalho, S. Oliva, A. Pereira and A. Rodriguez-Bernal, Attractors for Parabolic Problems with Nonlinear Boundary Conditions, Journal of Mathematical Analysis and Applications, 207:2 (1997).

[7] A. N. De Carvalho, J. W. Cholewa and T. Dlotko, Examples of global Attractors in parabolic Problems, Hokkaido Mathematical Journal, 27 (1998), pp. 77-108.

[8] J. W. Cholewa and T. Dlotko, Global Attractor for Sectorial Evolutionary Equations, Journal of Differential Equations, 125 (1996), pp. 27-39.

[9] T. Dlotko, Global Attractor for the Cahn-Hilliard Equation in $H^{2}$ and $H^{3}$, Journal of Differential Equations, 113 (1994), pp. 381-393.

[10] J. K. Hale, Asymptotic Behavior of Dissipative Systems, Mathematical Surveys and Monographs, 25 (1988), AMS.

[11] J. K. Hale And C. Rocha, Varying boundary conditions with large diffusivity, Journal de Mathématiques Pures et Appliquées, 66 (1987), pp. 139-158.

[12] D. Henry, Geometric Theory of Semilinear parabolic equations, Lecture Notes in Mathematics 840, Springer-Verlag, 1981.

[13] A. Lunardi, Analytic Semigroups and Optimal Regularity in Parabolic Problems, Progress in Nonlinear Differential Equations and Their Applications 16, Birkhäuser 1995.

[14] J. L. Lions And E. Magenes, Non homogeneous Boundary Value Problems and Applications I, Springer Verlag, 1961.

[15] O. A. Ladyzhenskaya, N. N. Ural'tseva and V. A. Solonnikov, Linear and Quasilinear Equations of Parabolic type, America Mathematical Society, Translations of mathemtical monographs 23, 1968.

[16] A. Rodríguez -Bernal, Sistemas Dinámicos Infinito Dimensionales, Aplicaciones a la Ecuación de Kuramoto - Velarde, Tesis Doctoral, Universidad Complutense de Madrid, 1991.

[17] A. Rodríguez -Bernal, Initial Value Problem and Asymptotic Low Dimensional Behaviour in the Kuramoto - Velarde Equation, Nonlinear Analysis, Theory, Methods \& Applications, 19:7 (1992), pp. 643-685.

[18] Existence, uniqueness and regularity of solutions of nonlinear evolution equations in extended scales of Hilbert Spaces, Center of Dynamical Systems and Nonlinear Studies, Report Series CDSNS91-61.

[19] - Ecuaciones de evolución semilineles, Curso 96-97, Lecture Notes Universidad Complutense de Madrid, 28040 Madrid, Spain.

[20] A. Rodríguez -Bernal And E. Zuazua, Parabolic Singular Limit of a Wave Equation with Localized Boundary Damping, Discrete and Continuous Dynamical Systems 1:3 (1996), pp. 
303-346.

[21] A. Rodríguez -Bernal and R. Willie, The Linear Parabolic Problem and Large Diffusion, to appear in Discrete and Continuous Dynamical Systems 2003.

[22] G. Sell And Y. You, Dynamics of Evolutionary equations, Springer Verlag, 2002.

[23] J. Smoller, Shock wave and Reaction-Diffusion Equations, Springer Verlag, 1983.

[24] R. Temam, Infinite Dimensional Dynamical Systems in Mechanics and Physics, Springer Verlag, New York 1988.

[25] R. Willie, Large Diffusivity Finite Dimensional Asymptotic Behaviour of a Semilinear Wave Equation, Journal of Applied Mathematics, 8 (2003), pp. 409-427, "Hindawi Publishing Coorporation".

[26] - Upper Semi-continuity of Attractors for Reaction and Diffusion System of equations in Sup- Norm, Abstract and Applied Analysis, 2003:00 (2003), pp. 1-25, "Hindawi Publishing Coorporation".

[27] - A semilinear system of reaction and difussion equations with nonlinear boundary conditions and large diffusion, to appear in Journal of Dynamics and Differential equations 2003.

[28] The Inertial Manifold for A Nonlinear Reaction-Diffusion System with Large Diffusion, submitted Journal of Differential Equations, April 2003. 